\title{
Supporting Information: FIB-SEM Tomography Probes the Meso-Scale Pore Space of an Individual Catalytic Cracking Particle
}

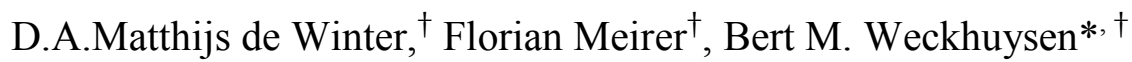 \\ ${ }^{\dagger}$ Inorganic Chemistry and Catalysis group, Debye Institute for Nanomaterials Science, Utrecht University, \\ Universiteitsweg 99, 3584 CG Utrecht, The Netherlands \\ *Corresponding author: b.m.weckhuysen@uu.nl
}

\section{Details of SEM images}

FCC particles were embedded in a resin ${ }^{1}$ and mechanically polished, resulting in disks of approximately $3 \mathrm{~cm}$ in diameter, one for the FCC1 catalyst particles and one for the FCC2 catalyst particles. The disks were mounted on an aluminum SEM stub with a cohesive carbon tape, with a small strip of carbon tape connecting the top surface with the aluminum stub to ensure electrical conductance. Subsequently, the two samples were coated with Pt/Pd (4 nm) in a Cressington HQ280 sputter coater.

The SEM images were recorded in a Nova Nanolab 600 Dualbeam (FEI, Eindhoven, The Netherlands), at $2 \mathrm{kV}, 0.21 \mathrm{nA}$, using the Through the Lens Detector (TLD) in combination with the immersion lens, in backscatter electron (BSE) mode. Individual FCC particles were imaged with a scan/pixel resolution of 5 $-6 \mathrm{~nm}$. The high scan resolution required the recording of multiple images per individual FCC particle. Stitching of the SEM images was done in Microsoft's Image Composite Editor (ICE).

The stitched images were segmented in Photoshop (CS5.1/version 12.1). First, the area surrounding an individual FCC particle was removed. Subsequently, the individual particle was selected and its 
histogram was normalized to a mean of approximately 126. Next, the noise in the image was removed by the Dust \& Scratches filter (conditions: Radius $=4$; Threshold $=0$ ). Finally, grey level thresholding (threshold value $=90$ ) was applied. The filter settings were established by visual inspection. To maintain consistency, all individual FCC particles are processed with the same values.

A script was written, which applied a grid of variable dimensions across an image of an individual FCC particle. The spatial porosity was determined as the ratio of the black pixels (pore space) over all pixels within the square. Pixels outside the FCC particle were neglected.

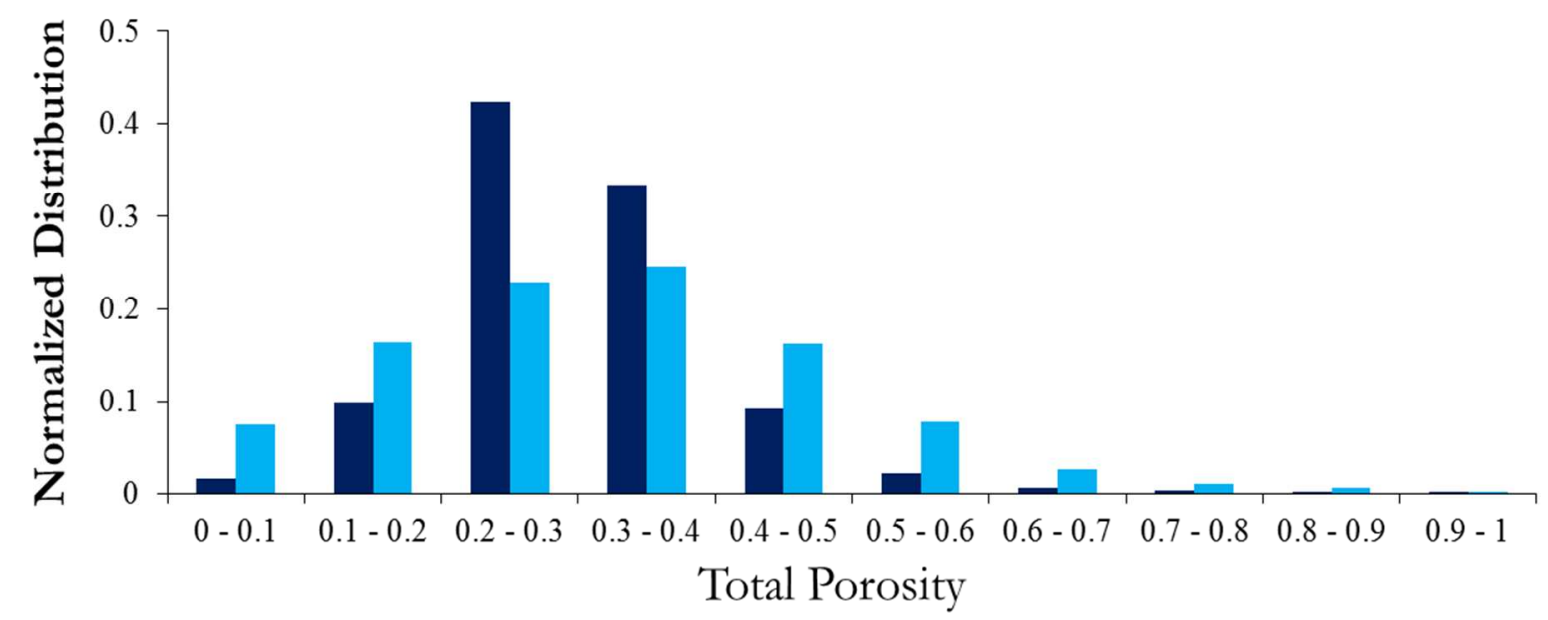

Figure S1: The spatial porosity distribution is based on the 2D SEM images, with grid dimensions of $2 \times 2$ $\mu \mathrm{m}^{2}$. FCC1 catalyst particles: Dark blue bars; FCC2 catalyst particles: Light blue bars.

\section{Details of FIB-SEM tomography}

The FCC catalyst particles were sprinkled on an aluminum SEM stub with a cohesive carbon sticker and subsequently coated with $\sim 4 \mathrm{~nm}$ Pt/Pd in a Cressington HQ280 sputter coater. Two SEM samples were prepared, one for FCC1 and one for FCC2. 
FIB - SEM Tomography was done in a Nova Nanolab 600 Dualbeam (FEI, Eindhoven, The Netherlands). The FIB - SEM Tomography routine is equivalent to most FIB - SEM Tomography routines described in the literature ${ }^{2}$, with the slices milled perpendicular to the surface. Prior to the routine itself, a protective layer of Pt deposition was deposited on top of the region of interest (ROI), with a thickness of approximately $1 \mu \mathrm{m}$. Trenches were milled alongside the ROI and a fiducial marker was created next to one of the trenches with the FIB. The fiducial marker is used by both the FIB and the SEM for alignment purposes. The final preparation step was milling a large cross section in front of the ROI.

The FIB conditions during the FIB-SEM Tomography series were $30 \mathrm{keV}, 0.3 \mathrm{nA}$. For FCC1 a total of 150 slices with dimensions (xyz) $35 \times 7 \times 0.02 \mu \mathrm{m}^{3}$ were milled and for FCC2 a total of 1000 slices with dimensions (xyz) $30 \times 7 \times 0.02 \mu \mathrm{m}^{3}$. Due to the more pronounced heterogeneity, the volume analyzed from FCC2 was chosen significantly larger.

After milling a slice, three SEM images were recorded alongside each other, from the resulting cross section, with a scan resolution of $2048 \times 1768$ and a horizontal field of view of $12.8 \mu \mathrm{m}$. SEM images were recorded in BSE mode with $2 \mathrm{kV}, 0.21 \mathrm{nA}$. The tilt angle of the cross section's surface with respect to the SEM imaging plane resulted in a vertical foreshortening of the images. In addition, the slice thickness exceeded the $X Y$ resolution by a factor of $\sim 3$. During further analysis, the resulting anisotropic voxel dimensions were maintained with dimensions of $6.26 \times 7.94 \times 20 \mathrm{~nm}^{3}(x, y, z)$ for both FCC1 and FCC2.

Due to computational limitations, two out of the three series of SEM images (from FCC1 and FCC2) were selected for further processing, based on overall image quality. The series of images were independently post-aligned using IMOD. ${ }^{3}$ The total volume available for further processing from FCC1 was two series of (xyz) $12.8 \times 7 \times 3 \mu \mathrm{m}^{3}$ and from FCC2 two series of (xyz) $12.8 \times 7 \times 20 \mu \mathrm{m}^{3}$. 
In the next step, sub-volumes with dimensions of $2 \times 2 \times 2 \mu \mathrm{m}^{3}$ were selected from the aligned series of images, considering any absence of distortion in the images due to local charging effects, and the images being well in focus. Each sub-volume consists of 100 images of $253 \times 320$ pixels. Due to the anisotropic voxel dimension, the sub-volumes are cubic in physical length scales.

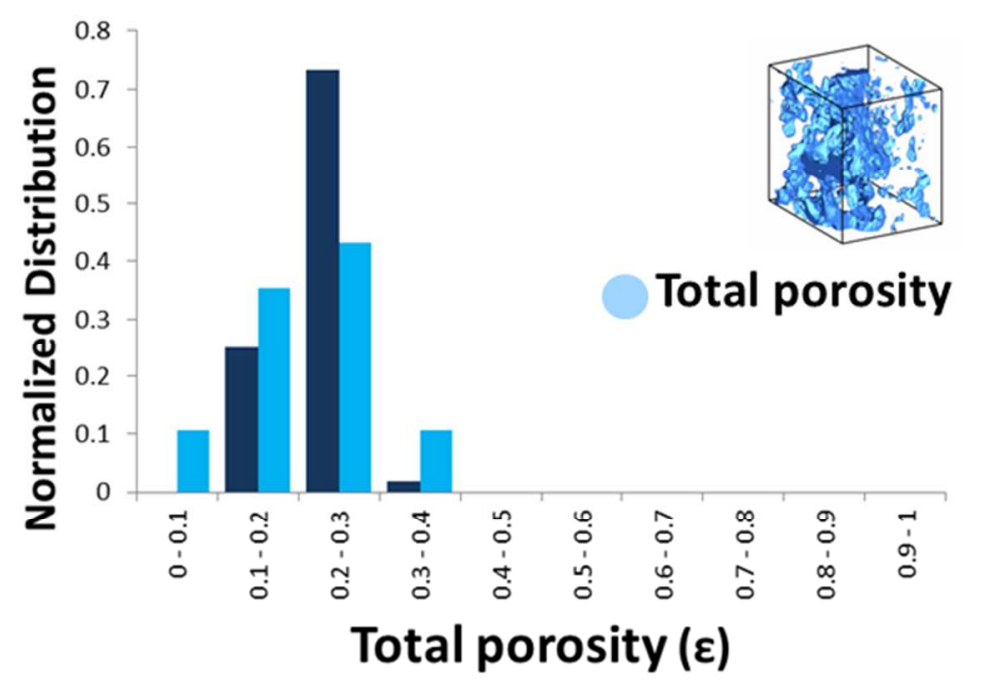

Figure S2: The porosity distribution of $50 \times$ FCC1 sub-volumes and $50 \times$ FCC2 sub-volumes. Due to the relatively small volume analyzed by FIB-SEM Tomography, the distributions are not as wide as the distributions determined from entire FCC catalyst particles (in 2D). Still, the FCC2 catalyst particle has a wider distribution than the FCC1 catalyst particle, in agreement with the observations and the 2D data, which are shown in Figure S1.

Further digital processing of the individual FCC sub-volumes was required, to distinguish the pore space from the solid phase. A routine was developed which is capable of automatically recognizing the pore space in $3 \mathrm{D}$ data sets. The routine combines grey level thresholding from the $2 \mathrm{D}$ images with grey level gradient thresholding in 3D. Due to the use of filters, the final voxel resolution is approximately 20 $\times 20 \times 20 \mathrm{~nm}^{3}$. 
The final step identified connected pore space from not-connected pore space. The assumption was made that mass transport occurs in one direction only, along the z-axis. Pore space in connection to both the entrance plane and the exit plane was defined as 'connected', whereas the remaining pore space was defined as 'not-connected'.

\section{Virtual volume algorithms}

Two algorithms were tested, a random algorithm and a random walk-like algorithm (referred to as 'snake' algorithm), generating virtual volumes of $50 \times 50 \times 50$ voxels. The random algorithm starts with an empty volume and replaces all pore voxels by a solid voxel with a probability equal to one minus the porosity of the sub-volumes. The 'snake algorithm', starts with a solid volume. An $\mathrm{x}$ and y coordinate is chosen randomly as starting position, while the z coordinate is set to zero. The selected voxel is assigned as pore voxel. A path of pore voxels is created by taking successive steps from the starting position onwards, with step sizes of $-1 \geq \Delta x \geq 1 ;-1 \geq \Delta y \geq 1$; $-1 \geq \Delta z \geq 1$, all with equal probabilities. The boundaries perpendicular to the plane $A_{0}$ (Figure 2, main text) are periodic, allowing the path to continue at the opposite planes. The length $L_{0}$, along the z-axis, is temporarily increased by $0.5 \mathrm{~L}_{0}$ in both directions, providing the potential for a homogeneous porosity distribution throughout the virtual volume. The pathway is restricted to a z-coordinate value of $-0.5 L_{0}$. Once that coordinate is reached, a randomly generated step of $\Delta z=-1$ is overruled by $\Delta z=0$. When $z=0.5 L_{0}$ is reached, the pore generation is finished. Finally, the connected porosity was established of the virtual volumes, similar to the FCC sub-volumes. Note that the random algorithm allows for generating a virtual volume with a porosity value close to a pre-determined value. Porosity generated by the snake algorithm can only be evaluated after the sub-volume has been created. The periodic boundaries allows for generating not- 
connected pore spaces when applying the snake algorithm, although Figure $5 c$ shows a tendency for virtual volumes towards a higher connectivity $\left(\varepsilon_{\text {connected }} \approx \varepsilon_{\text {total }}\right)$ than for the FCC sub-volumes.

\section{Percolation Probability}

A percolating volume (percolation $=1$ ) contains a pathway from the entrance to the exit plane. Percolation has been determined for 10.000 virtual volumes generated with the snake algorithm. The percolation values ( 1 or 0 ) have been plotted against the porosity of the corresponding virtual volumes. Subsequently, a curve is fitted to the moving average $(\Delta \varepsilon=0.2)$ of the scatter plot. The average percolation values equal the percolation probability for a virtual volume with a corresponding porosity. Accurate fitting of the curve between $\varepsilon=0.09$ and $\varepsilon=0.39$ was improved by plotting the square root of the percolation probability. For $\varepsilon>0.39$, the percolation probability is 1 , while the percolation probability below $\varepsilon=0.09$ drops to zero very fast. The implementation in the script: $\operatorname{PP}(\varepsilon<0.07)=0$, while the fitted equation (Figure 6a) is used for $0.07 \leq \varepsilon<0.39$. 


\section{Example for the developed up-scaling procedure}
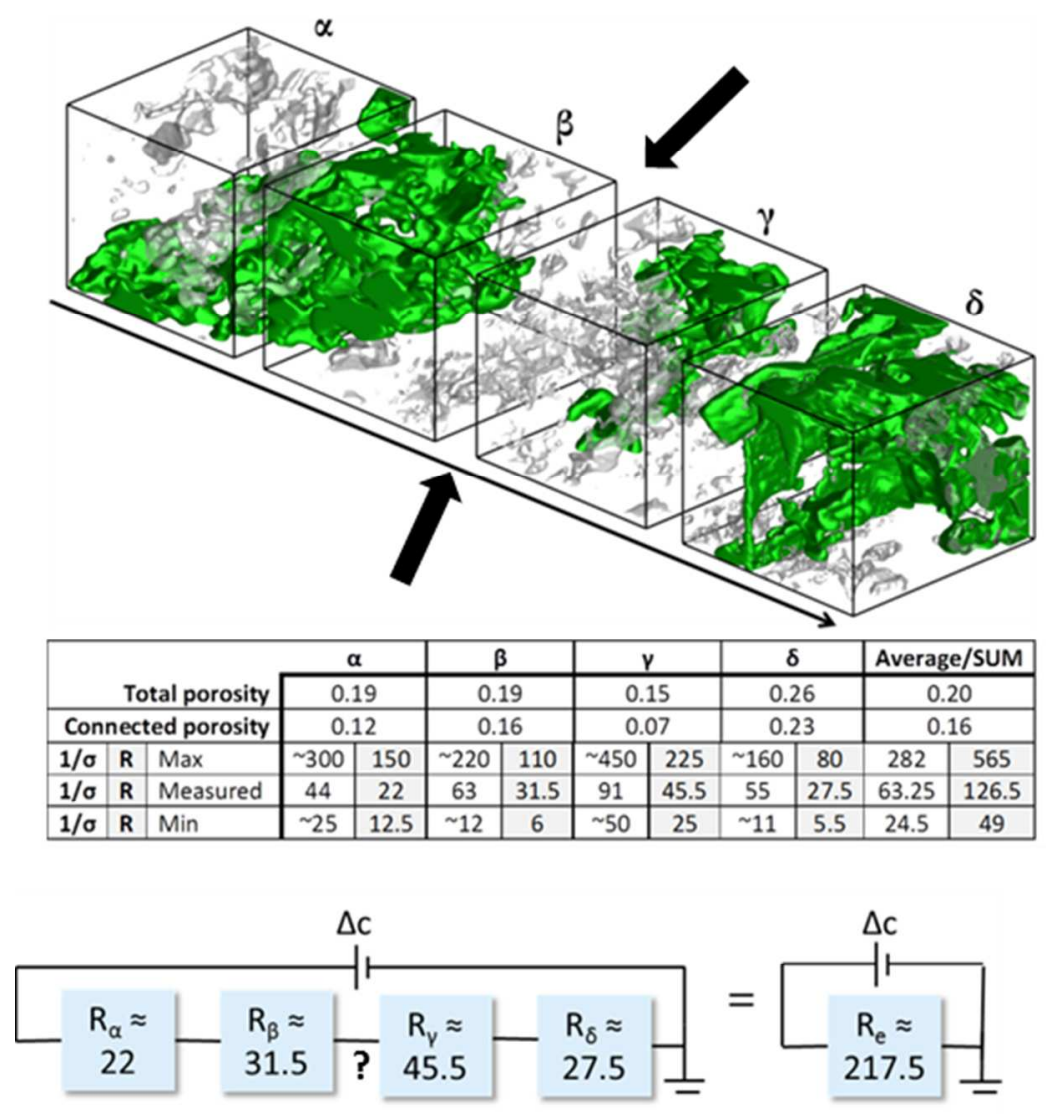

Figure S3: The inverse transport ability is determined for each FCC sub-volume separately. From the inverse transport ability $\left(\sigma^{-1}\right)$, the transport resistance $R$ is calculated. Following the analogue with the electrical resistance, the four resistors are placed in series, resulting in an equivalent resistance value. The first assumption, continuity of the pore space from one FCC sub-volume to another, is valid. The second assumption, placing percolating volumes in series which results in another percolating volume, is not valid for this particular example. The connection between FCC sub-volumes $\beta$ and $\gamma$ does not exist. As a result, the series $\alpha-\delta$ is not percolating, resulting in $R=\infty$. In case of a 3D arrangement, percolation becomes more likely, as many (detouring) pathways are likely to exist. 


\section{Fitting of the probability distribution function}

Table S1: The numerical details of the probability distribution function $F\left(\sigma^{-1}\right)$ where $\sigma^{-1} \sim N\left(a, b^{2}\right)$, for Figure $6 a$ in the main text of the manuscript. The values for $\varepsilon_{\text {connected }}=1$ are added as theoretical limits.

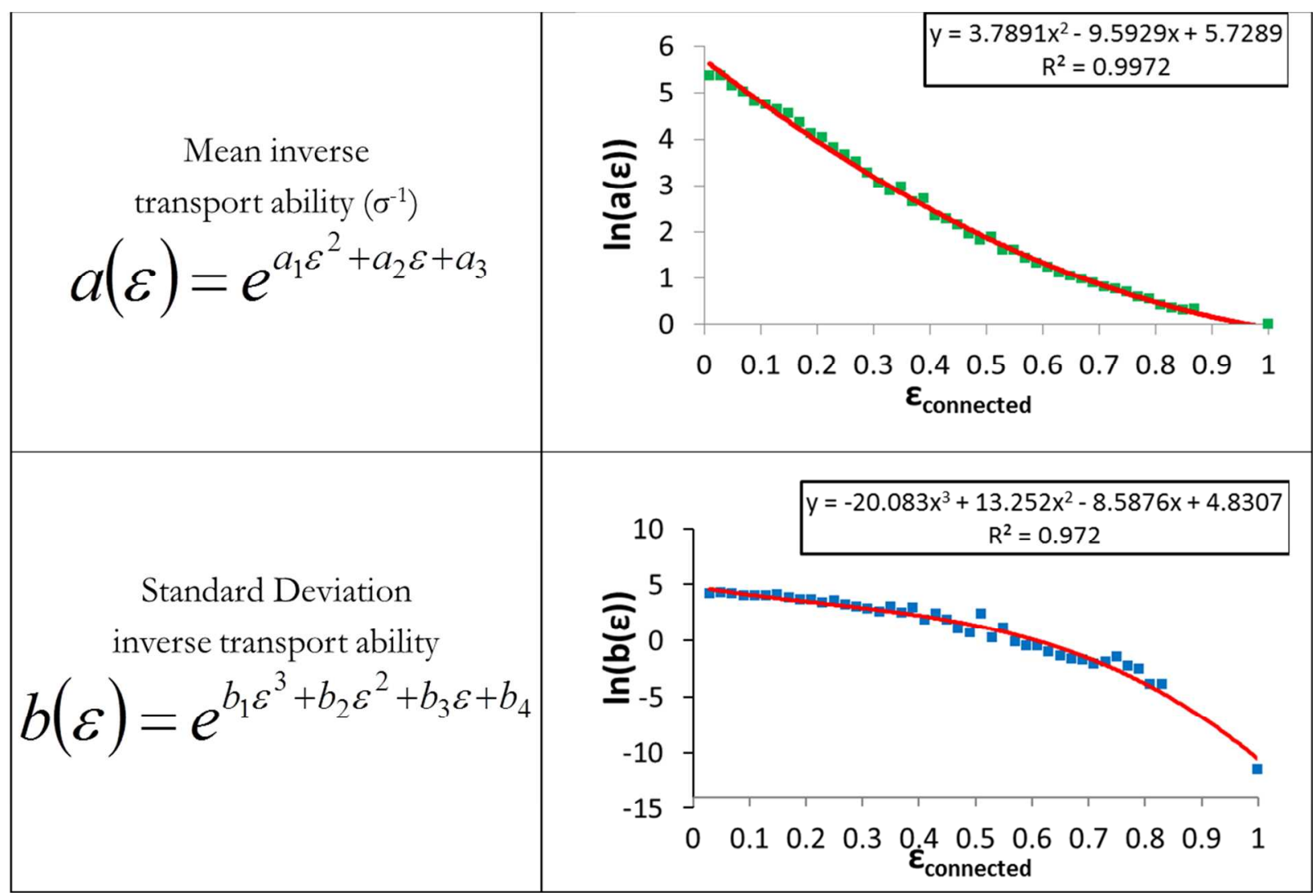




\section{Up-scaling parameters}

Table S2: The parameters used for the up-scaling routine for the FCC1 and FCC2 catalyst particles.

\begin{tabular}{|c|c|c|c|c|c|c|c|c|}
\hline \multicolumn{4}{|c|}{ Mean inverse transport ability: } & & \multicolumn{4}{|c|}{$a(\varepsilon)=a_{1} \varepsilon^{a_{2}}$} \\
\hline \multicolumn{4}{|c|}{$\begin{array}{c}\text { Standard Deviation } \\
\text { inverse transport ability }\end{array}$} & & \multicolumn{4}{|c|}{$b(\varepsilon)=b_{1} e^{b_{2} \varepsilon}$} \\
\hline & FCC1 & & & & FCC2 & & & \\
\hline & $8 \times 8 \times 8$ & & & & $8 \times 8 \times 8$ & & & \\
\hline & Mean & & St Dev & & Mean & & St Dev & \\
\hline & a1 & a2 & b1 & b2 & a1 & a2 & b1 & b2 \\
\hline Particle 1 & 0.776 & -2.954 & 1425.5 & -19.31 & 0.518 & -3.398 & 8140.5 & -22.94 \\
\hline Particle 2 & 0.524 & 3.246 & 4581.5 & -23.72 & 0.454 & -3.518 & 8174.2 & -22.69 \\
\hline Particle 3 & 0.5651 & -3.141 & 6252 & -26.36 & 0.409 & -3.523 & $6.00 \mathrm{E}+06$ & -57.73 \\
\hline Particle 4 & 0.7286 & -3.022 & 18927 & -32.3 & 0.545 & -3.358 & 3882.5 & -18.91 \\
\hline \multirow[t]{4}{*}{ Particle 5} & 0.4735 & -3.257 & 16617 & -31.2 & 0.335 & -3.752 & 8890.8 & -23.09 \\
\hline & $32 \times 32 \times 32$ & & & & $32 \times 32 \times 32$ & & & \\
\hline & Mean & & St Dev & & Mean & & St Dev & \\
\hline & a1 & a2 & b1 & b2 & a1 & a2 & b1 & b2 \\
\hline Particle 1 & 0.727 & -3.015 & 26447 & -30.6 & 0.49 & -3.411 & 3537.6 & -22.58 \\
\hline Particle 2 & 0.4995 & -3.295 & 12004 & -29.64 & 0.405 & -3.525 & 13101 & -27.27 \\
\hline Particle 3 & 0.7362 & -3.063 & 194089 & -47.95 & 0.16 & -4.149 & $6.00 \mathrm{E}+06$ & -57.73 \\
\hline Particle 4 & 0.2818 & -3.697 & 71540 & -40.34 & 0.514 & -3.378 & 15219 & -25.72 \\
\hline \multirow[t]{4}{*}{ Particle 5} & 0.1929 & -3.888 & 183920 & -44.59 & 0.322 & -3.688 & 15927 & -27.94 \\
\hline & $96 \times 96 \times 96$ & & & & $96 \times 96 \times 96$ & & & \\
\hline & Mean & & St Dev & & Mean & & St Dev & \\
\hline & a1 & a2 & b1 & b2 & a1 & $a 2$ & b1 & b2 \\
\hline Particle 1 & 0.7935 & -2.947 & 265468 & -40.4 & 0.6 & -3.213 & 15942 & -31.26 \\
\hline Particle 2 & 0.7786 & -2.941 & 28728 & -36.71 & 0.765 & -2.977 & 17084 & -30.92 \\
\hline Particle 3 & 1.0317 & -2.887 & 16020 & -35.04 & 1.346 & -2.521 & 7048.2 & -27.89 \\
\hline Particle 4 & 2.6973 & -2.312 & 10481 & -34.75 & 1.127 & -2.726 & 2810.9 & -24.17 \\
\hline Particle 5 & 0.5191 & -3.196 & 12728 & -34.35 & 0.678 & -3.085 & 28348 & -33.63 \\
\hline
\end{tabular}


8. The up-scaling results

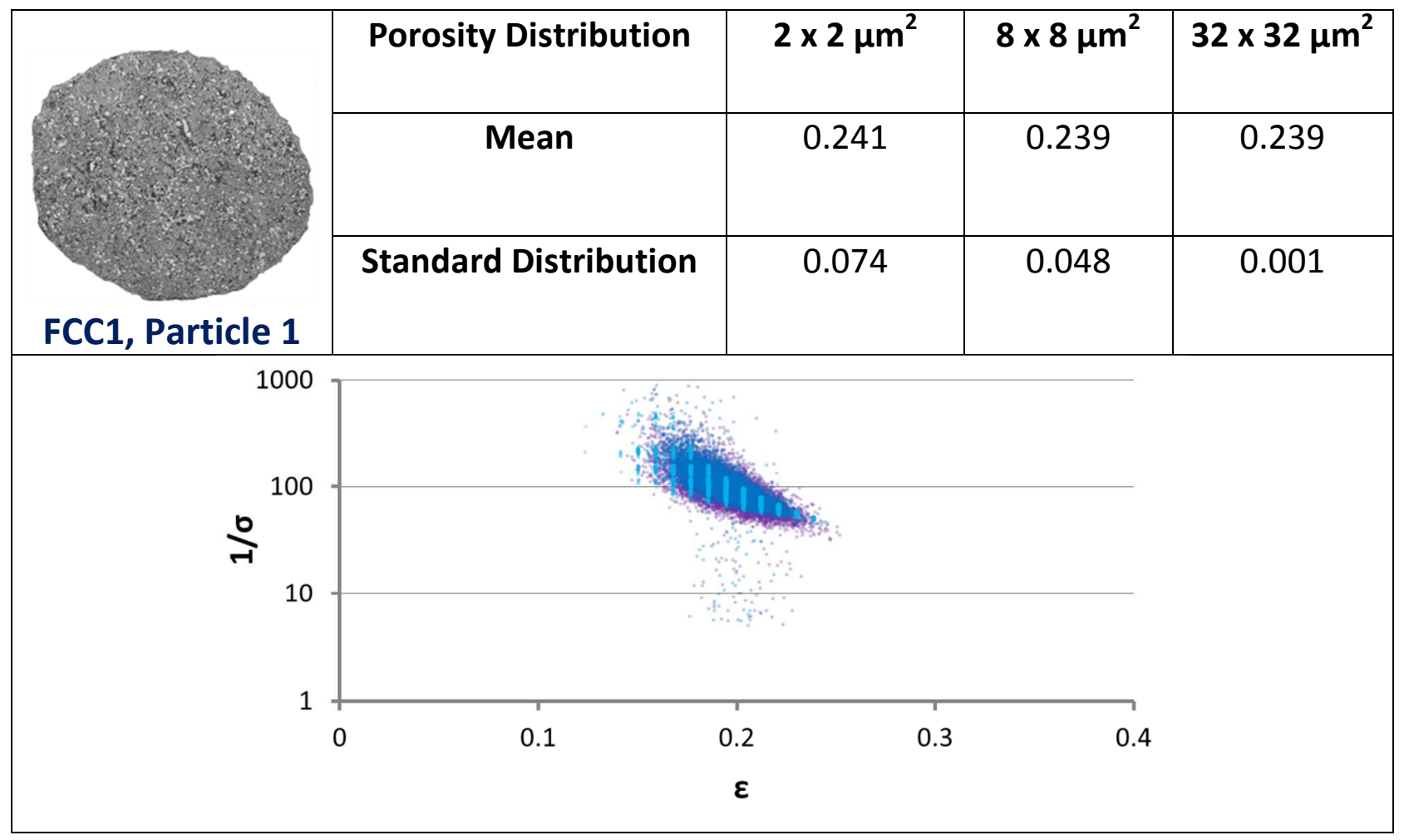

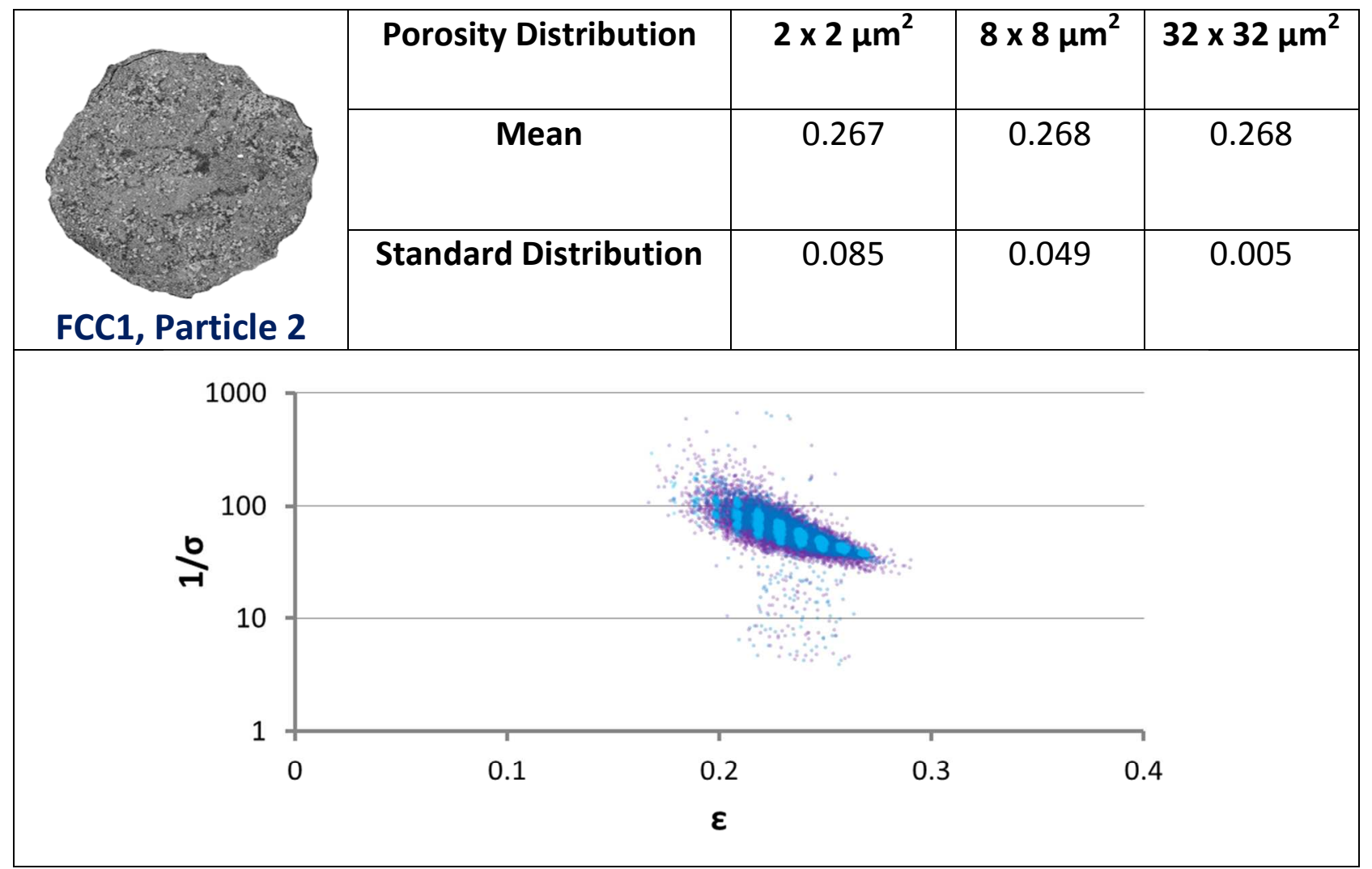




\begin{tabular}{|c|c|c|c|c|}
\hline & Porosity Distribution & $2 \times 2 \mu \mathrm{m}^{2}$ & $8 \times 8 \mu m^{2}$ & $32 \times 32 \mu \mathrm{m}^{2}$ \\
\hline & Mean & 0.323 & 0.323 & 0.323 \\
\hline FCC1, Particle 3 & Standard Distribution & 0.072 & 0.040 & 0.008 \\
\hline 1000 & & & & \\
\hline 100 & & & & \\
\hline 10 & & & & \\
\hline & 0.1 & 0.2 & 0.3 & 0.4 \\
\hline & & $\varepsilon$ & & \\
\hline
\end{tabular}

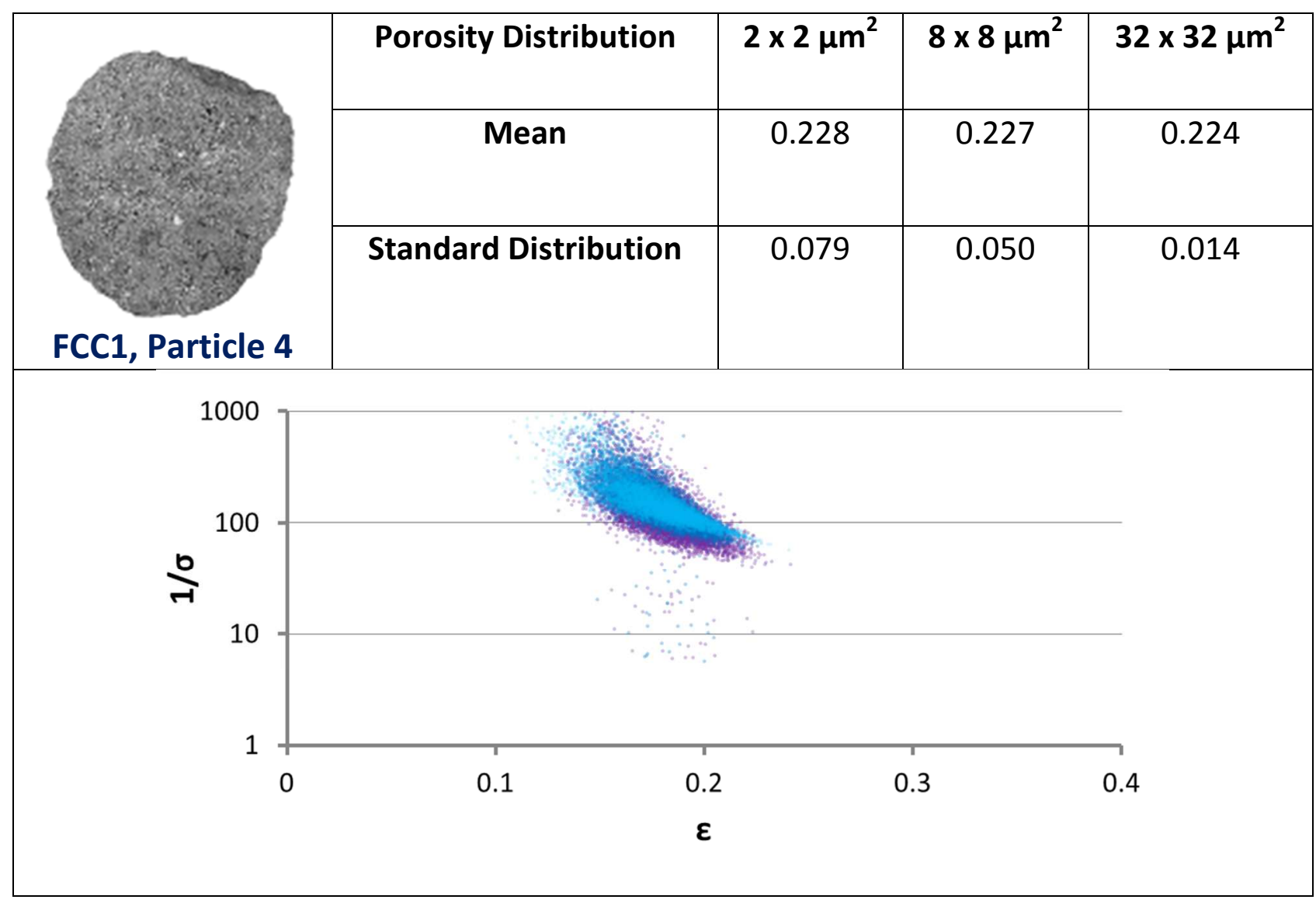




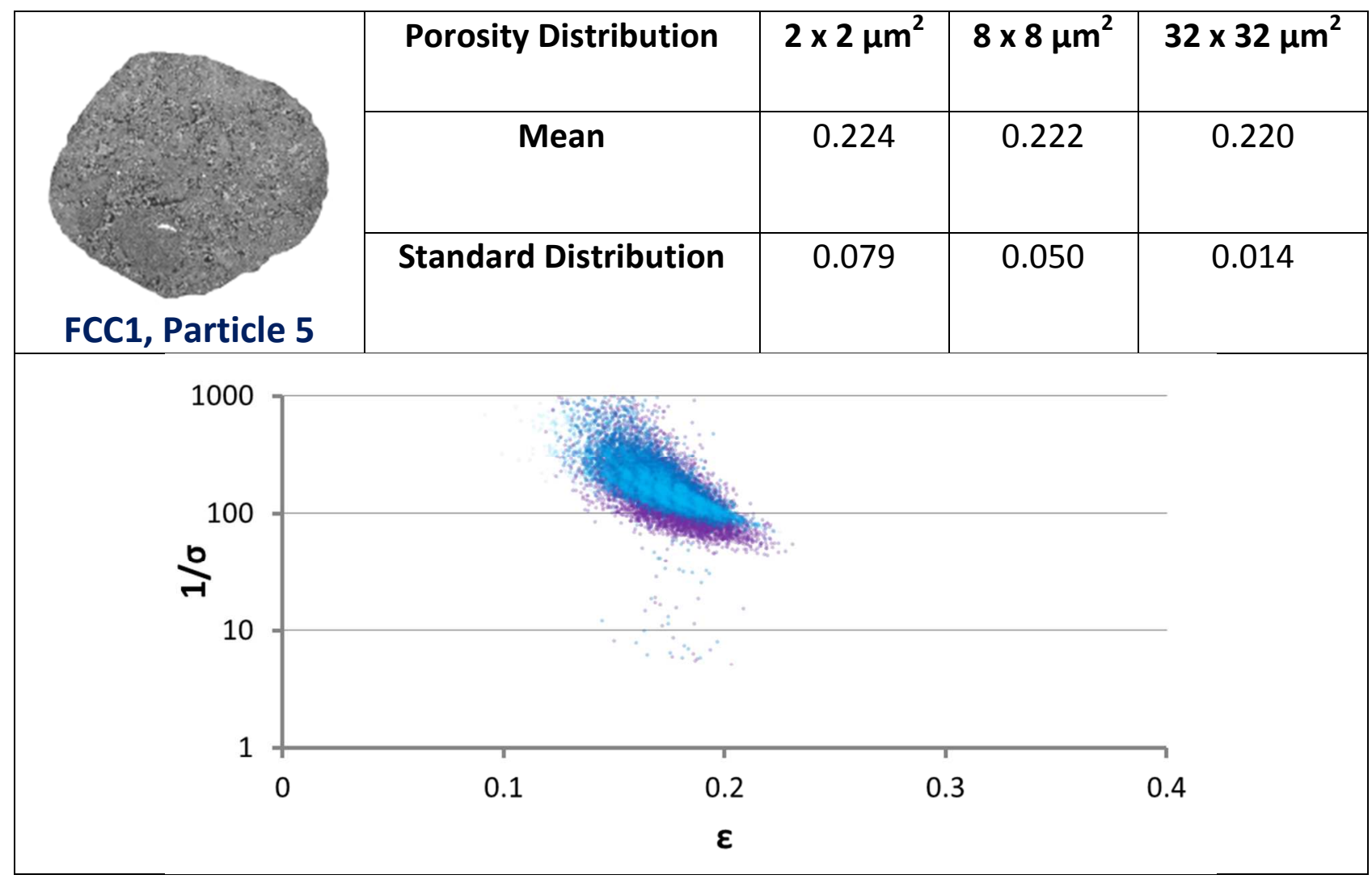

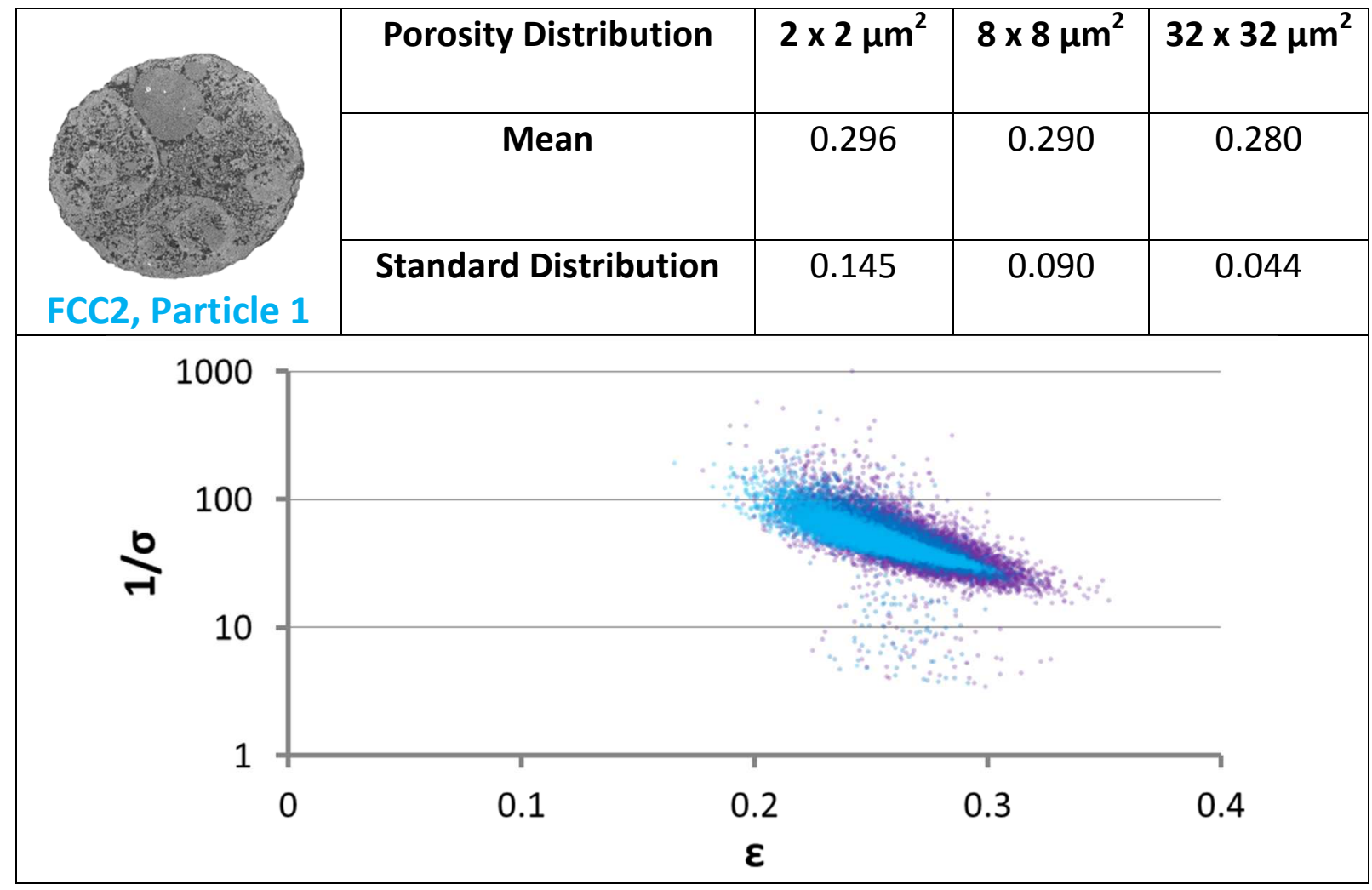




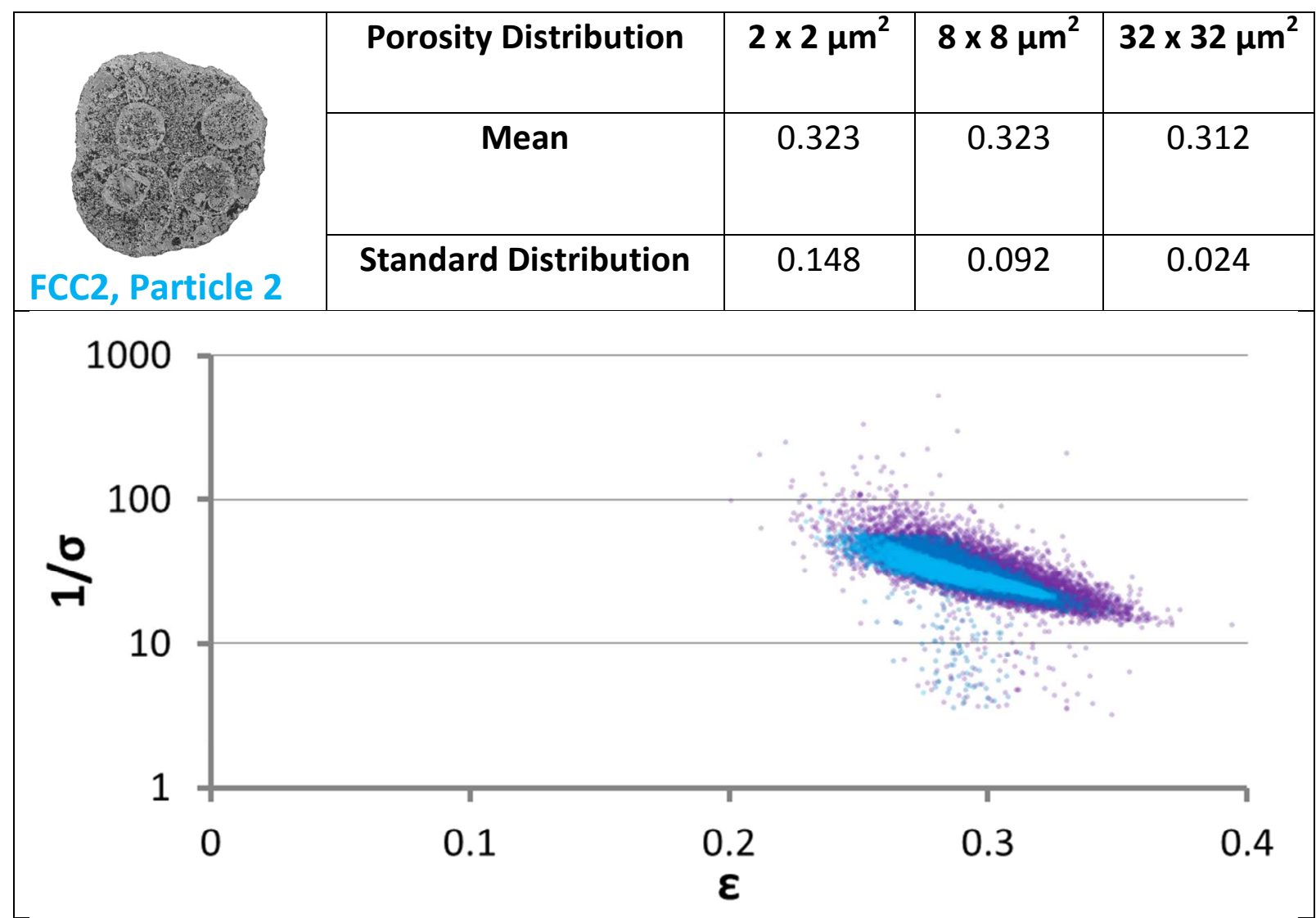

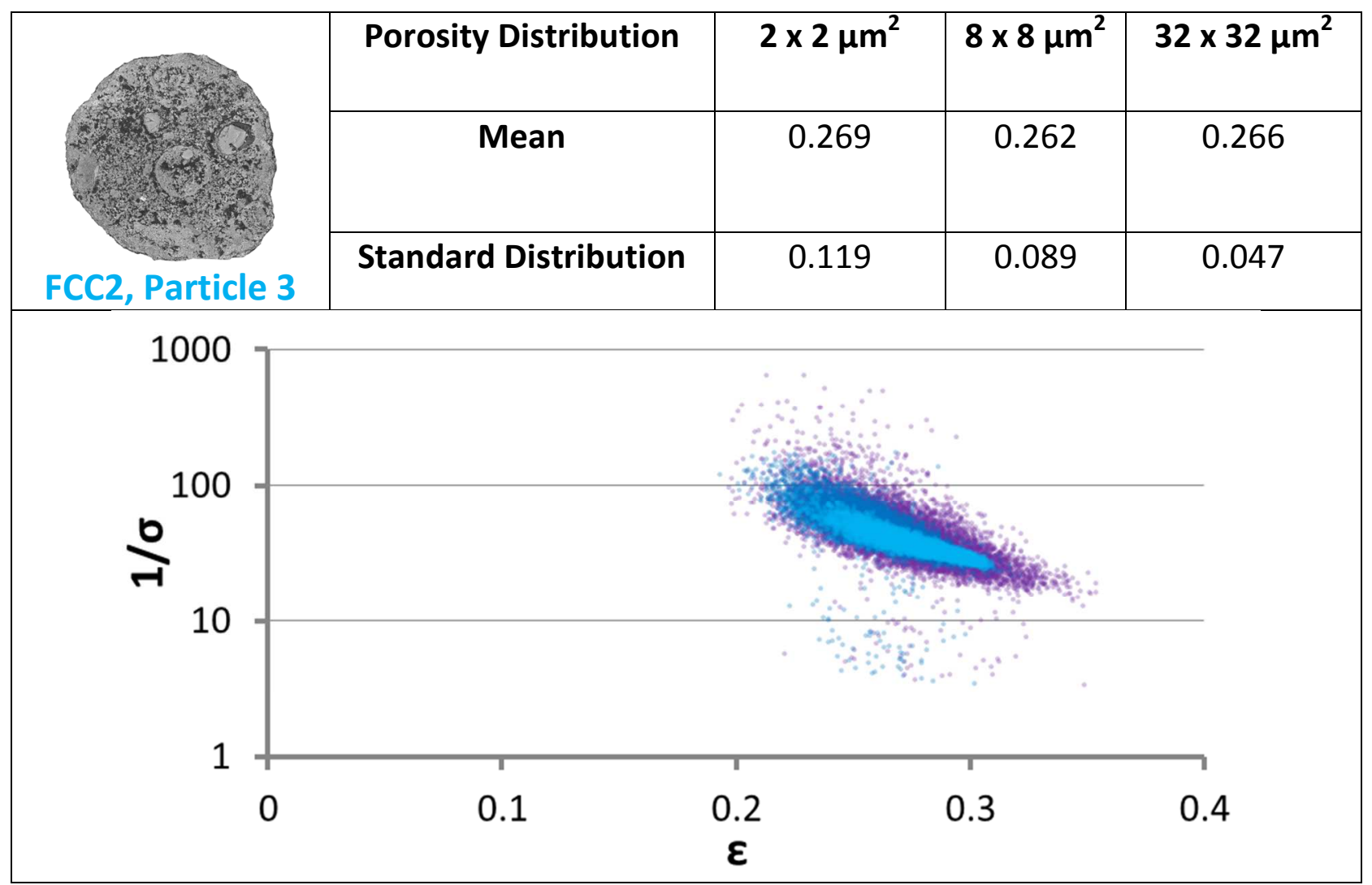




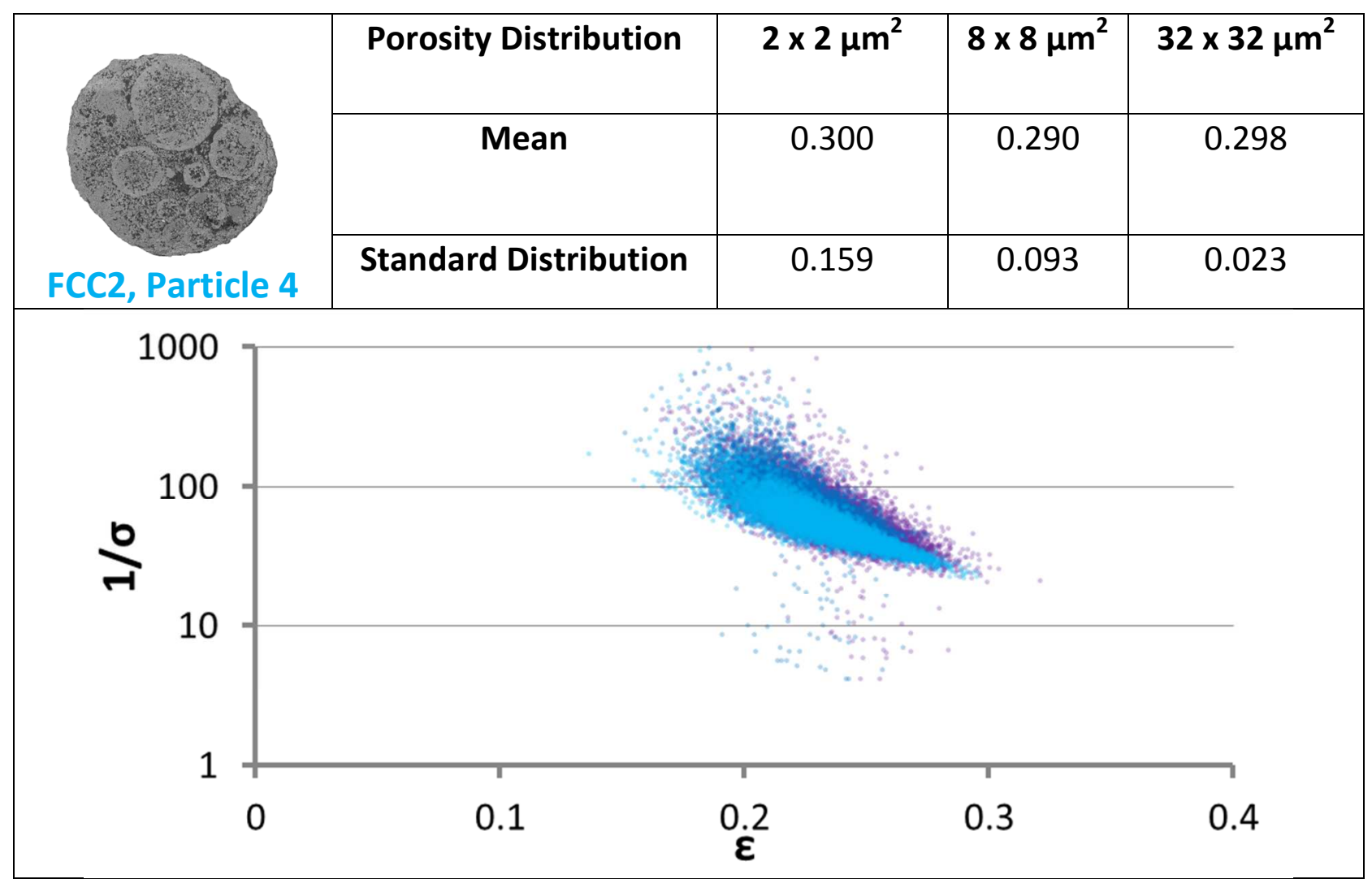

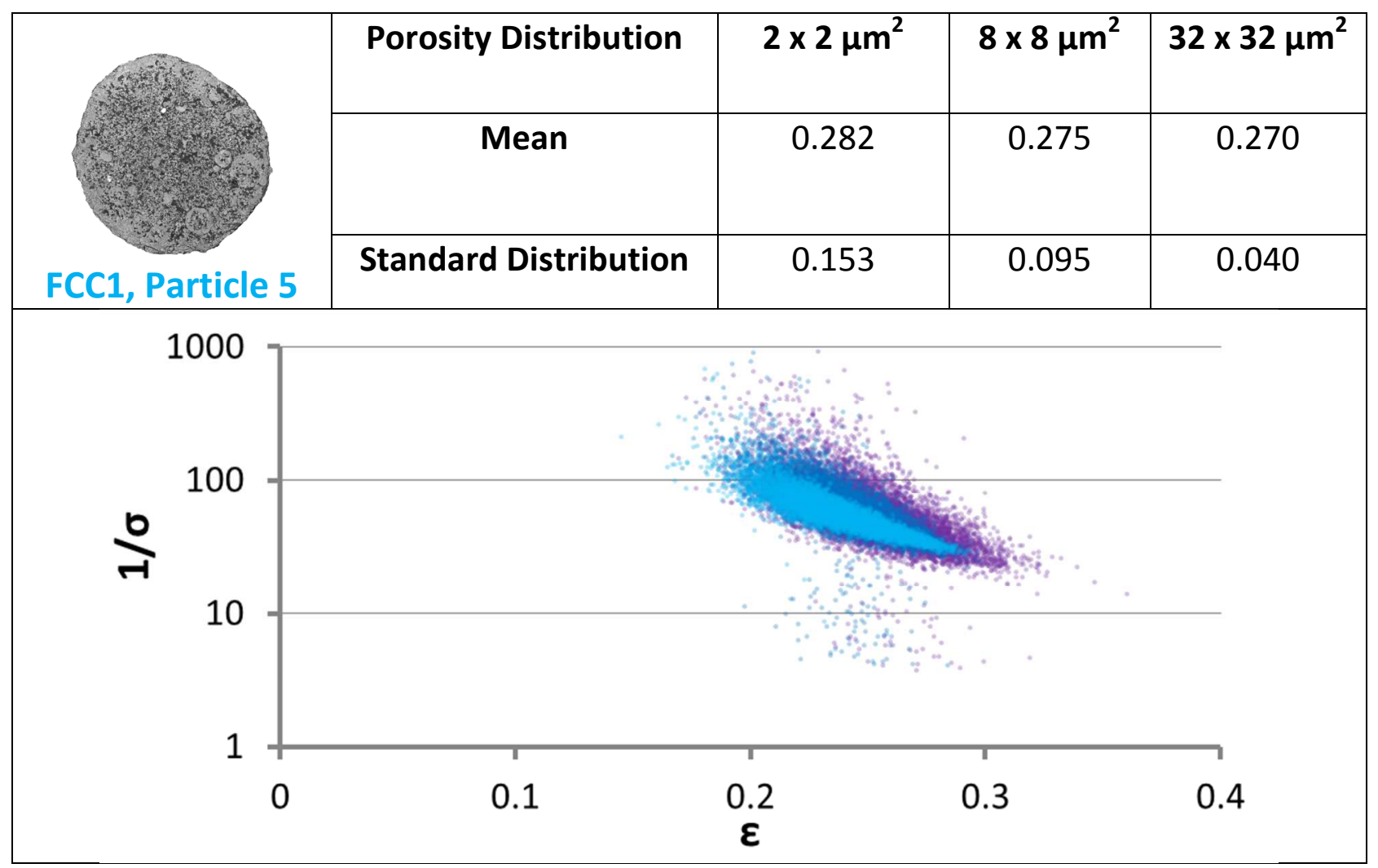


Figure S4 (pages S10 - S14): The results of the up-scaling routine split for the five FCC1 and FCC2 catalyst particles under study.

\section{References}

(1) Karreman, M. A.; Buurmans, I. L. C.; Geus, J. W.; Agronskaia, A. V.; Ruiz-Martínez, J.; Gerritsen, H. C.; Weckhuysen, B. M. Angew. Chem. Int. Ed. 2012, 51, 1428-1431.

(2) Karwacki, L.; De Winter, D. A. M.; Aramburo, L. R.; Lebbink, M. N.; Post, J. A.; Drury, M. R.; Weckhuysen, B. M. Angew. Chem. Int. Ed. 2011, 50, 1294-1298.

(3) Kremer, J. R.; Mastronarde, D. N.; McIntosh, J. R. J. Struct. Biol. 1996, 116, 71-76. 